\title{
Rapid and complicated HIV genotype expansion among high-risk groups in Guangdong Province, China
}

Ping-Ping Zhou', Guolong Yu², Yi-Qun Kuang ${ }^{1,3}$, Xu-He Huang ${ }^{1}$, Yan Li ${ }^{2}$, Xiaobing Fu ${ }^{2}$, Peng Lin ${ }^{2}$, Jin Yan ${ }^{2 *}$ and Xiang $\mathrm{He}^{1 *}$ (D)

\begin{abstract}
Background: Guangdong Province is one of the most developed and populous provinces in southern China, with frequent foreign exchanges and large transient population. The annual number of cases of HIV/AIDS reported in Guangdong has been higher than most of provinces in China for several successive years. HIV infection by heterosexual transmission occurs across the province, with transmission among men who have sex with men occurring mainly in larger urban centers. There is a lack of widespread and representative data on the distribution of HIV subtypes in Guangdong. This study aimed to thoroughly investigate and estimate the prevalence and distribution of HIV-1 subtypes using a city-based sampling strategy to better understand the characteristics of HIV transmission in Guangdong.
\end{abstract}

Methods: Archived plasma samples $(n=1205)$ from individuals diagnosed as HIV-1 infection in 2013 were selected randomly from all 21 cities in Guangdong Province. Genotypes were determined using env and/or gag sequences using phylogenetic analysis. The distributions of HIV genotypes in different risk groups and different cities were analyzed.

Results: A total of 15 genotypes, including six discordant genotypes, were identified. The four main HIV-1 subtypes in Guangdong were CRF01_AE (43.2\%), CRF07_BC (26.3\%), CRF55_01B (8.5\%), and CRF08_BC (8.4\%). CRF01_AE was the predominant subtype in all risk populations. The high mobility of people shaped the complexity of the HIV genotypes, while the switch of risk factors affected the distribution and future trend of HIV-1 genotypes in Guangdong. Another epicenter located in the western region in addition to the known epicenter cities in the Pearl River Delta region of Guangdong may exist.

Conclusions: Our study provides a comprehensive molecular epidemiologic dataset to understand the diversity and distribution of HIV genotypes in Guangdong, as well as to clarify the unique region- and risk group-specific transmission dynamics. The results provide critical and insightful information for more effective intervention strategies to limit HIV transmission in the future.

Keywords: HIV-1, Genotype, Risk factor, Molecular epidemiology, Guangdong, China

\footnotetext{
* Correspondence: yanjin212@qq.com; xhe.2013@gmail.com

${ }^{2}$ Guangdong Provincial Center for Disease Control and Prevention, 160

Qunxian Road, Panyu District, Guangzhou 511430, China

${ }^{1}$ Guangdong Provincial Institute of Public Health, Guangdong Provincial

Center for Disease Control and Prevention, 160 Qunxian Road, Panyu District,

Guangzhou 511430, China

Full list of author information is available at the end of the article
}

(c) The Author(s). 2019 Open Access This article is distributed under the terms of the Creative Commons Attribution 4.0 International License (http://creativecommons.org/licenses/by/4.0/), which permits unrestricted use, distribution, and reproduction in any medium, provided you give appropriate credit to the original author(s) and the source, provide a link to the Creative Commons license, and indicate if changes were made. The Creative Commons Public Domain Dedication waiver (http://creativecommons.org/publicdomain/zero/1.0/) applies to the data made available in this article, unless otherwise stated. 


\section{Background}

Guangdong Province (hereafter referred to as Guangdong) is located on the southern coast of China. It was one of the first provinces to establish relationships with the outside world, starting in 1978. The first imported case of human immunodeficiency virus (HIV) was identified in 1986 in Guangdong. Local HIV cases were reported in injection drug users (IDU) in 1996, with a subsequent rapid spread among drug users, with the number of cases reaching a peak in 2006 [1-3]. The sexual transmission of HIV began to increase in 2005 and by 2009 this route had become the predominant means of transmission, exceeding transmissions via IDU. It is noteworthy that HIV cases in men who have sex with men (MSM) accounted for over $10 \%$ of the infected population by 2013 [3]. The switch of major risk factors may have affected the distribution of HIV subtypes in Guangdong.

Before 2003, there were four prevalent HIV-1 genotypes in Guangdong-CRF01_AE, CRF07_BC, CRF08_BC, and subtype B-with CRF07_BC and CRF01_AE found mainly in IDU, CRF01_AE in cases of sexual transmission, and subtype B in blood donors and transfusion recipients [1, 4]. The examination of clinical samples in 2008 and 2009 studies revealed similar genotype distribution in Guangdong, with the exceptions of the newly detected subtype C, CRF02_AG, and discordant genotypes $[5,6]$. Two studies in Guangdong focused on the HIV genotypes for different risk populations in the cities of as Guangzhou [7] and Shenzhen [8]. A recent publication described the genotype distribution and dynamics in HIV infected MSM in Shenzhen, and reported the complex HIV genotypes and emergence of new recombinants in this at-risk population [9]. However, the foregoing studies either involved limited sample sizes or focused on a specific location or risk population, and thus are not representative of the HIV genotype distribution across Guangdong.

Here we report a comprehensive study using a citybased sampling strategy to explore the geographic and demographic distribution of HIV genotypes across Guangdong. We estimated the likely prevalence and distribution of HIV-1 genotypes in Guangdong using the genotypic data of 1205 archived specimens combined with HIV case report data of various risk groups in prefecture-level cities in Guangdong. Our results provide a comprehensive dataset on the characteristics and diversity of HIV and acquired immunodeficiency syndrome (AIDS) in Guangdong.

\section{Methods}

\section{Study participants and sampling strategy}

A total of 1205 plasma specimens were randomly selected using the Random Sampling function in Excel software from HIV-1 positive samples previously collected and archived by Guangdong Provincial Center for Disease Control and Prevention, based on the reported case number of each city in Guangdong in 2013 (Table 1). The epidemiological data of the patients, including their risk group, gender, age, ethnicity, marital status, and education, were acquired from China Information System for Disease Control and Prevention. There were no significant differences in the risk groups between genotyped samples and reported cases for each city (all $p>0.05$ using the $\chi^{2}$ test, Additional file 1: Table S1).

\section{RNA extraction, amplification, and sequencing}

Viral RNA was extracted from $200 \mu \mathrm{l}$ of plasma using the MagPure Viral Nucleic Acid KF Kit (Magen, Guangzhou, China) with a KingFisher Flex Purification System (Thermo Fisher Scientific, Waltham, MA, USA) following the manufacturer's instructions. Both gag (HXB2: 781-1836 nt) and env (HXB2: 7002-7645 nt) fragments were amplified using the primers and amplification conditions as previously described [10] with the PrimeScript One Step RT-PCR Kit and Premix Taq (Takara Bio, Dalian, China). PCR products were sent to a commercial company (Zixi Biotech, Beijing, China) for sequencing.

\section{Sequence analysis and subtype determination}

The HIV sequences were aligned using the HIVAlign tool [6] (https://www.hiv.lanl.gov/content/sequence/VIRALIGN/viralign.html) and then merged with HIV-1 group $M$ subtyping references from Los Alamos HIV Sequence Database using BioEdit [11]. HIV-1 genotypes were determined based on phylogenetic analysis. Neigh bor-joining phylogenetic trees were reconstructed with the Kimura 2-parameter substitution model and evaluated by the bootstrap method with 1000 replicates using MEGA 5.2 [12]. The recombinants were screened by the RIP 3.0 tool [13].

The HIV-1 genotype of each patient was determined based on the genotypes of both gag and env fragments. If only one gene fragment was available, the genotype of that gene was assigned. Samples with different genotypes identified from gag and env regions were deemed discordant genotypes and were assigned gag/env genotype designations, such as CRF01/C.

\section{Estimation of HIV genotypes in different cities and risk groups}

The HIV genotypes in different prefecture cities and risk groups were estimated according to previously described methods [6]. Briefly, the basic analysis unit in our study was a specific risk population in a city. The number of cases for each genotype in the unit was estimated by multiplying the proportion of that genotype in the unit with the total number of HIV cases reported in the unit. 
Table 1 HIV case reported in Guangdong in 2013 and sample selection

\begin{tabular}{|c|c|c|c|c|c|c|}
\hline City & $\begin{array}{l}\text { Number of } \\
\text { reported cases }\end{array}$ & $\begin{array}{l}\text { Number of selected } \\
\text { samples }\end{array}$ & $\begin{array}{l}\text { Sampling } \\
\text { Ratio }\end{array}$ & $\begin{array}{l}\text { Number of } \\
\text { genotyped samples }\end{array}$ & $\begin{array}{l}\text { Corrected number of represented } \\
\text { cases in the study }\end{array}$ & $\begin{array}{l}\text { Representation in } \\
\text { the study }\end{array}$ \\
\hline Guangzhou & 1342 & 81 & $6.0 \%$ & 77 & 1330 & $5.8 \%$ \\
\hline Shenzhen & 1189 & 80 & $6.7 \%$ & 65 & 1187 & $5.5 \%$ \\
\hline Dongguan & 579 & 76 & $13.1 \%$ & 67 & 575 & $11.7 \%$ \\
\hline Foshan & 482 & 76 & $15.8 \%$ & 61 & 481 & $12.7 \%$ \\
\hline Jiangmen & 291 & 68 & $23.4 \%$ & 60 & 291 & $20.6 \%$ \\
\hline Yangjiang & 187 & 90 & $48.1 \%$ & 58 & 183 & $31.7 \%$ \\
\hline Zhongshan & 186 & 64 & $34.4 \%$ & 60 & 186 & $32.3 \%$ \\
\hline Zhanjiang & 174 & 127 & $73.0 \%$ & 55 & 174 & $31.6 \%$ \\
\hline Huizhou & 155 & 59 & $38.1 \%$ & 59 & 154 & $38.3 \%$ \\
\hline Yunfu & 149 & 59 & $39.6 \%$ & 45 & 147 & $30.6 \%$ \\
\hline Maoming & 147 & 59 & $40.1 \%$ & 57 & 137 & $41.6 \%$ \\
\hline Qingyuan & 146 & 59 & $40.4 \%$ & 59 & 143 & $41.3 \%$ \\
\hline Zhaoqing & 108 & 53 & $49.1 \%$ & 50 & 108 & $46.3 \%$ \\
\hline Shantou & 91 & 50 & $54.9 \%$ & 49 & 88 & $55.7 \%$ \\
\hline Zhuhai & 81 & 48 & $59.3 \%$ & 48 & 81 & $59.3 \%$ \\
\hline Shaoguan & 61 & 31 & $50.8 \%$ & 31 & 60 & $51.7 \%$ \\
\hline Heyuan & 48 & 33 & $68.8 \%$ & 33 & 48 & $68.8 \%$ \\
\hline Meizhou & 47 & 30 & $63.8 \%$ & 29 & 45 & $64.4 \%$ \\
\hline Jieyang & 40 & 20 & $50.0 \%$ & 20 & 38 & $52.6 \%$ \\
\hline Shanwei & 24 & 20 & $83.3 \%$ & 18 & 23 & $78.3 \%$ \\
\hline Chaozhou & 21 & 22 & $104.8 \%$ & 20 & 21 & $95.2 \%$ \\
\hline Total & 5548 & 1205 & $21.7 \%$ & 1021 & 5500 & $18.6 \%$ \\
\hline
\end{tabular}

For a unit in which no specimen was obtained, the number of reported cases was excluded from the calculations. The adjusted totals and corresponding sampling ratios for each city are listed in the last two columns of Table 1. The estimated number of persons infected with each genotype in each risk group across the province was obtained by summing the units across all cities. Similarly, the estimated proportions and numbers of individuals infected with each genotype in each region and the province were obtained by summing up the units accordingly. The prevalence of HIV-1 genotypes was calculated by weighting the sampled prevalence with the size of the corresponding risk group in any given city; if no specimen was obtained for the risk- and city-specific subunit, the case count for that subunit was removed from the analysis. Approximately $0.9 \%$ (48 of 5548) reported cases were removed from the total case number (Table 1 ).

\section{Results}

Distribution of HIV-1 genotypes in Guangdong

A total of 1205 plasma specimens were selected from diagnosed HIV-positive persons, which constitute
$21.7 \%$ of the total reported HIV-1 cases in 2013 in Guangdong. Among the samples, 1021 were determined for the HIV-1 genotypes from the 1.1-kb gag and/or the 563-bp env regions (Table 1). $862 \mathrm{gag}$ genotypes $(71.5 \%$ of samples) and 900 env genotypes (74.7\%) were acquired, and both gag and env genotypes were assessable for 741 samples (61.5\%).

Nine major HIV-1 subtypes and circulating recombinant forms (CRF) in total were detected in this study. They included subtypes B, B' (Thai B strain), C, G, CRF01_AE, CRF07_BC, CRF08_BC, CRF55_01B, and CRF59_01B (Fig. 1a and Additional file 2: Table S2). CRF01_AE (43.2\%), CRF07_BC (26.3\%), CRF55_01B (8.5\%), and CRF08_BC (8.4\%) were the predominant HIV-1 subtypes circulating in Guangdong (Fig. 1a). These four subtypes were responsible for $86.4 \%$ of reported HIV infections in Guangdong in 2013. Minor HIV-1 genotypes included CRF59_01B (1.7\%), B (1.1\%), B'(0.7\%), C (0.9\%), and G (0.3\%). They were classified as "other" genotypes. The remaining $8.8 \%$ were discordant genotypes between gag and env fragments, and included CRF01/C (4.1\%), CRF07/CRF01 (3.1\%), B/CRF01 (0.6\%), CRF01/B 


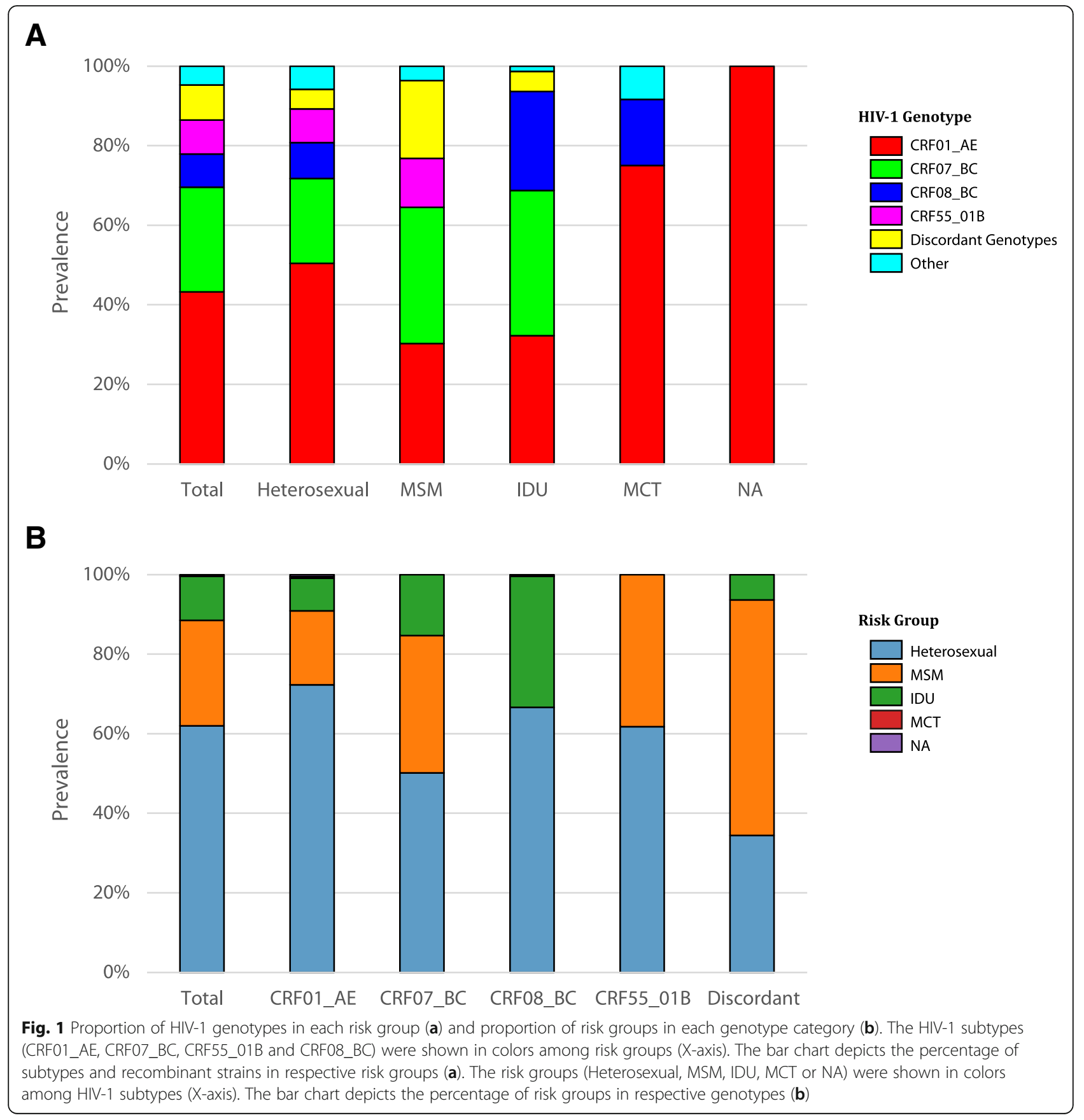

(0.5\%), 0107/CRF01 (0.4\%), and BC/C (0.04\%) (Additional file 2: Table S2).

\section{HIV-1 genotype distribution by risk group}

As illustrated in Fig. 1b, sexual transmission including heterosexual and MSM was the primary risk factor in Guangdong, which accounted for $88.4 \%$ (61.9\% for heterosexual and $26.5 \%$ for MSM) of HIV reported cases in 2013, followed by IDU including sexual plus IDU (11.1\%) and mother to child transmission (MCT, 0.2\%).
About $0.2 \%$ of HIV cases resulted from unknown transmission routes.

CRF01_AE (50.5\%), CRF07_BC (21.3\%), CRF08_BC (9.0\%), and CRF55_01B (8.5\%) were the main HIV-1 subtypes circulating among heterosexuals, accounting for $89.3 \%$ of infections in this risk group, followed by discordant genotypes (4.9\%) (Fig. 1a). The greatest genotype diversity was identified among heterosexuals, in which almost all the HIV-1 genotypes were detected, except B/CRF01. The main genotypes prevailing among MSM were CRF07_BC (34.2\%), CRF01_AE (30.3\%), and 
CRF55_01B (12.3\%). Interestingly, CRF08_BC was not detected in MSM, while the discordant genotypes accounted for $19.6 \%$ of MSM infections, which was the highest ratio in all risk groups (Fig. 1a). Other genotypes in MSM were subtype B (1.8\%), CRF59_01B (1.6\%), and $\mathrm{B}^{\prime}(0.3 \%)$. Three main genotypes were detected in IDU, including CRF07_BC (36.5\%), CRF01_AE (32.2\%), and CRF08_BC (24.9\%), followed by subtype C (1.3\%) and discordant genotypes (5.1\%). Only three HIV-1 genotypes, including CRF01_AE (75.0\%), CRF08_BC (16.7\%), and subtype $\mathrm{C}(8.3 \%)$, were detected among MCT.

\section{HIV-1 genotype distribution by geographic region}

According to the social-economic status, Guangdong could be classified into four regions: Pearl River Delta (PRD) region, eastern, western, and northern. The PRD region located in the central part of Guangdong has a prevalent Cantonese culture and is one of the most densely urbanized areas in the world, which includes the cities of Guangzhou, Shenzhen, Dongguan, Foshan, Jiangmen, Zhongshan, Huizhou, Zhaoqing, and Zhuhai. The eastern Guangdong is Teochew dialect-based and includes the four prefecture cities of Jieyang, Chaozhou, Shantou, and Shanwei. Northern Guangdong is dominated by mountains and includes four cities (Meizhou, Heyuan, Shaoguan, and Qingyuan). The Hakka dialects is prevalent in these cities. Western Guangdong is Cantonese culture based and includes the four cities of Zhanjiang, Maoming, Yangjiang, and Yunfu. As illustrated in Fig. 2, most HIV-1 cases in Guangdong were distributed in the PRD region (79.9\%), followed by west (11.6\%), north (5.4\%), and east (3.1\%) of Guangdong.

The estimated city-specific distributions of HIV-1 genotypes are presented in Fig. 2. In the PRD region, heterosexual (56.6\%), MSM (31.8\%), and IDU (11.6\%) were the major risk groups. The PRD region comprised the highest genotype diversity, and almost all the genotypes, except $\mathrm{BC} / \mathrm{C}$, were identified in this region. CRF01_AE

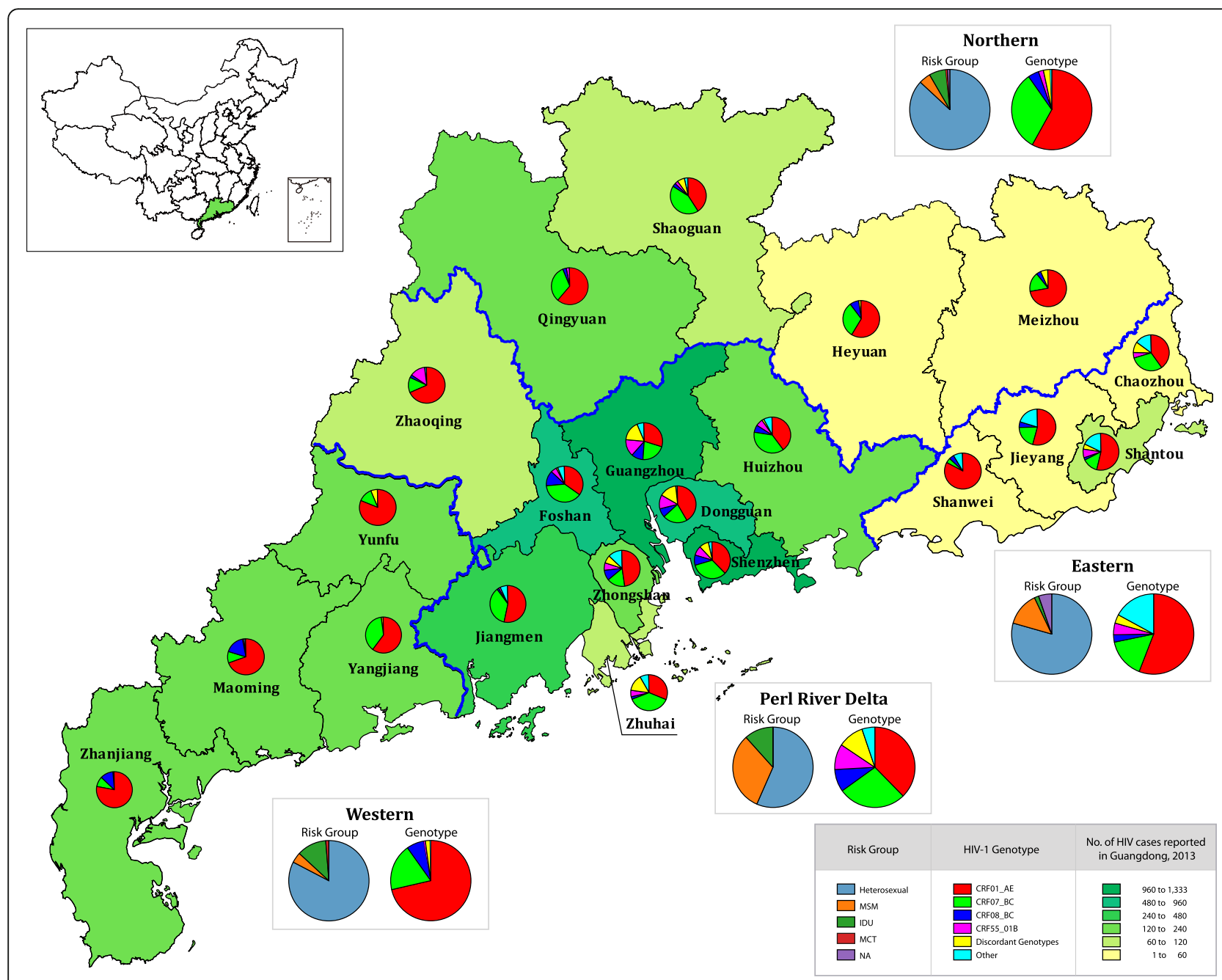

Fig. 2 Distribution of HIV-1 genotypes in different cities of Guangdong. It is illustrated based on the dataset tabulated in Additional file 2: Table S2 
(37.7\%), CRF07_BC (27.4\%), CRF55_01B (10.3\%), and discordant genotypes $(10.4 \%)$ were the main genotype categories. Most of the cases of CRF55_01B (453 of 471, $96.2 \%$ ) and discordant genotypes (459 of $485,94.6 \%$ ) were found in this region. Heterosexuals $(60.9 \%)$ and MSM (39.1\%) were the main risk groups for CRF55_01B, while MSM (62.3\%) and heterosexuals (31.6\%) were the main risk group for discordant genotypes.

In the western region, heterosexuals (82.7\%) accounted for the majority of HIV-1 cases, followed by IDU (11.9\%), MSM (4.2\%), and MCT (1.3\%). CRF01_AE (71.4\%), CRF07_BC (18.8\%), and CRF08_BC (7.0\%) were the main prevailing HIV-1 strains, followed by CRF55_01B (0.6\%), discordant genotypes $(1.9 \%$; $1.4 \%$ for CRF07/CRF01 and 0.5\% for 0107/CRF01), and CRF59_01B (0.3\%). Over 70\% of HIV cases were infected with CRF01_AE in heterosexual (72.8\%), MSM (74.1\%), and MCT (75\%) risk groups. The CRF01_AE composition ratio in the western region was the highest in all geographic regions, although the proportion of heterosexuals in the western region $(82.7 \%)$ was less than that in the northern region (86.9\%).

In the northern region, heterosexual (86.9\%) was the predominant risk group, followed by IDU (6.7\%), MSM (4.7\%), and not available (NA, 1.3\%). CRF01_AE (58.1\%) and CRF07_BC (32.2\%) were the main HIV-1 genotypes, followed by CRF08_BC (4.4\%), CRF55_01B (2.0\%), and discordant genotypes $(2.7 \%$; $1.3 \%$ for CRF07/CRF01, $1.0 \%$ for $0107 / C R F 01$, and $0.3 \%$ for CRF01/C). Lower composition ratios for CRF01_AE were found in heterosexuals (60.6\%), MSM (28.6\%), and IDU (35\%), while higher CRF07_BC ratios were found in those three risk groups.

In the eastern region, heterosexuals (79.4\%) and MSM (13.5\%) were the main risk groups, followed by IDU (1.8\%) and NA (5.3\%). CRF01_AE (55.9\%), CRF07_BC (15.9\%), and other genotypes (17.1\%; $8.8 \%$ for subtype B, $5.3 \%$ for $\mathrm{B}^{\prime}$, and $2.9 \%$ for subtype $\mathrm{C}$ ) were the main genotype categories in this region, followed by CRF55_01B (4.7\%), CRF08_BC (2.9\%), and discordant genotypes (3.5\%; $1.2 \%$ for CRF07/CRF01, $1.2 \%$ for BC/C, $0.6 \%$ for CRF01/ $\mathrm{C}$, and $0.6 \%$ for $0107 / \mathrm{CRF} 01)$. Among all geographic regions, the highest proportion of other genotypes category was observed in both heterosexuals $(17.0 \%$; $6.7 \%$ for subtype $\mathrm{B}, 6.7 \%$ for $\mathrm{B}^{\prime}$, and $3.7 \%$ for subtype $\mathrm{C}$ ) and MSM (26.1\%; all for subtype B) in the region. Of note, although the least number of HIV cases were reported in this region, there were 11 HIV-1 genotypes detected, which was the second highest number of genotypes.

\section{Distribution of HIV-1 strains in Guangdong by city and risk group}

The geographic distributions of the main HIV-1 genotype categories in Guangdong are illustrated in Fig. 3. CRF01_AE was detected in all 21 cities accounting for
43.2\% of reported HIV infections in Guangdong in 2013, which accounted for the highest proportion of HIV genotypes among 18 cities, except for Foshan, Zhuhai, and Shaoguan. The majority (69.7\%) of CRF01_AE was distributed in the seven cities of Shenzhen (18.7\%), Guangzhou (16.9\%), Dongguan (10.1\%), Foshan (7.0\%), Jiangmen (6.5\%), Zhanjiang (5.7\%), and Yunfu (5.0\%). The estimated number of CRF01_AE cases in Zhanjiang was $43.6 \%$ higher than that in the neighboring city of Maoming. It is conceivable that Zhanjiang was another possible epicenter for CRF01_AE in Guangdong along with Shenzhen and Guangzhou in the PRD region. The major risk groups for CRF01_AE were heterosexuals (72.3\%) and MSM (18.6\%), followed by IDU (8.3\%) (Fig. 1b).

CRF07_BC was also detected in all cities in Guangdong. The majority (83.5\%) of CRF07_BC infections were observed in the PRD region, especially in the five cities of Shenzhen (26.7\%), Guangzhou (18.7\%), Foshan (12.7\%), Dongguan (8.5\%), and Jiangmen (7.4\%). Heterosexuals (50.1\%), MSM (34.5\%), and IDU (14.2\%) were the main risk groups for CRF07_BC (Fig. 1b). Similar to CRF01_AE, the estimated number of CRF07_BC in Zhanjiang was higher than its nearby cities.

CRF55_01B was identified in 15 of 21 cities; the exceptions of were Yangjiang, Yunfu, Heyuan, Meizhou, Jieyang, and Shanwei. Most of the CRF55_01B cases were detected in the PRD region (96.2\%) primarily in Guangzhou (42.9\%), Shenzhen (22.5\%), Dongguan (15.1\%), and Foshan (5.5\%). The two risk factors for CRF55_01B infections were heterosexual (61.8\%) and MSM (38.2\%).

CRF08_BC was detected in 18 cities (not in Yangjiang, Yunfu, and Chaozhou). The majority (83.5\%) of CRF08_BC infections were identified in the PRD region $(86.3 \%)$ and the western region $(9.8 \%)$, primarily in Guangzhou (29.9\%), Shenzhen (24.1\%), Foshan (14.3\%), Dongguan (10.0\%), and Maoming (5.2\%). Heterosexuals (66.6\%) and IDUs (32.3\%) were the primary risk populations for CRF08_BC infections (Fig. 1b), and no CRF08_BC was identified in MSM population.

Discordant genotypes were detected in 14 cities. Most (94.6\%) were distributed in the PRD region, especially in Guangzhou (48.2\%), Shenzhen (20.4\%), and Dongguan (18.4\%). MSM (59.2\%) and heterosexuals (34.4\%) were the main risk factors for the discordant genotypes in Guangdong, followed by IDU (6.4\%).

CRF59_01B, subtypes B and B', and subtypes C and G accounted for $35.5,24.0,15.3,18.7$ and $6.5 \%$ of the other genotypes, respectively. Most CRF59_01B infections were identified in the PRD region (97.8\%) including Foshan (32.3\%), Guangzhou (18.3\%), Shenzhen (16.1\%), Jiangmen (10.8\%), Dongguan (9.7\%), Zhongshan (4.3\%), Zhuhai (4.3\%), and Huizhou (2.2\%), as well as the Maoming (2.2\%) in the western region. CRF59_01B 


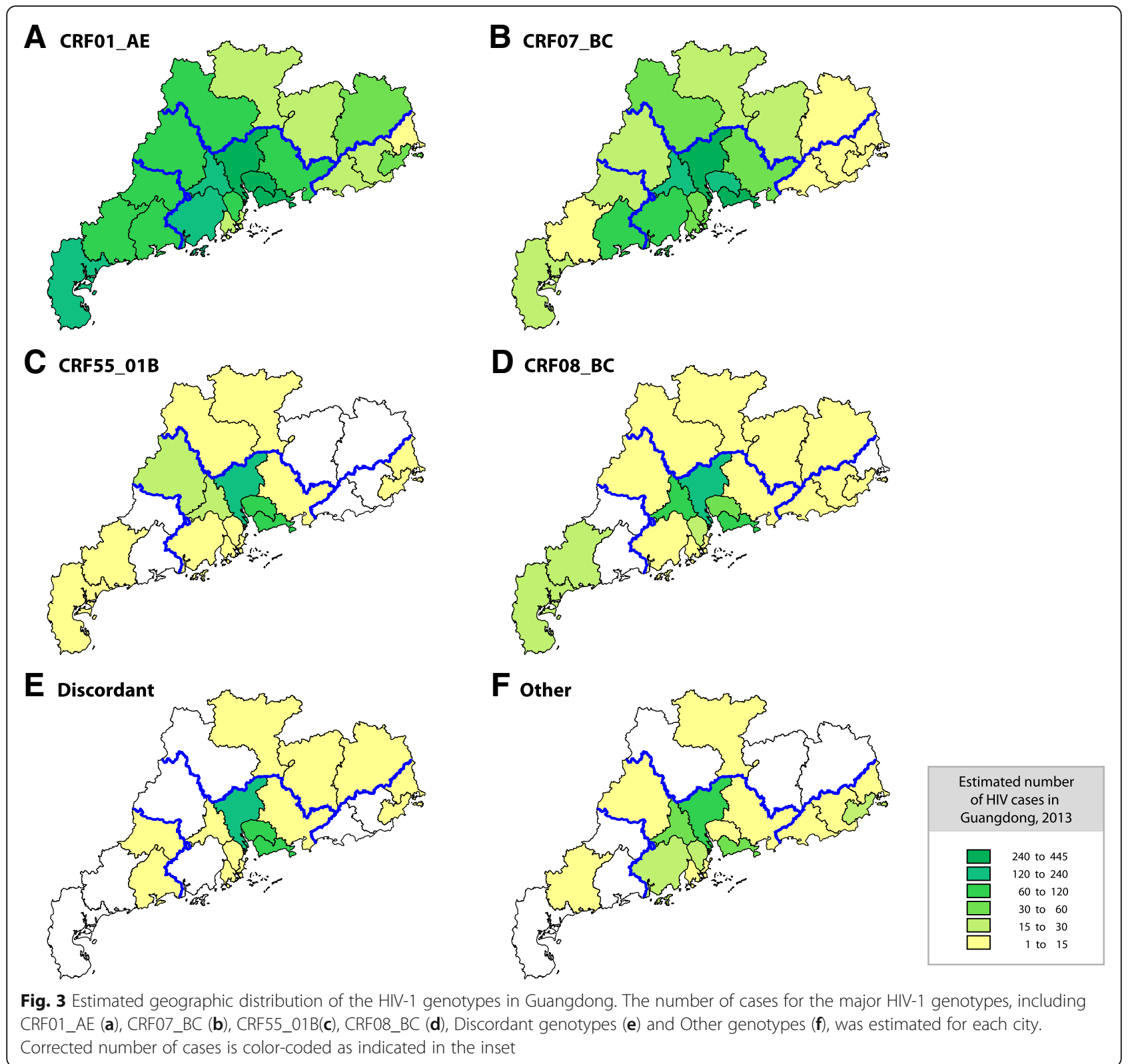

circulated in heterosexual (75.3\%) and MSM (24.7\%) groups. Subtypes B and B' were identified in the PRD region (76.2 and $72.5 \%$, respectively), the eastern region ( 23.8 and $22.5 \%$, respectively), and the northern region (5.0\% for $\left.\mathrm{B}^{\prime}\right)$. Heterosexuals comprised $58.7 \%$ of people with strain B and $90 \%$ of those with strain B', whereas $41.3 \%$ of B and $10 \%$ of $B^{\prime}$ were MSM. Subtype C was identified mainly in the PRD region $(89.8 \%)$, including Guangzhou (34.7\%), Jiangmen (20.4\%), Zhongshan (20.4\%), Huizhou (10.2\%), and Zhaoqing (4.1\%). There were also $\mathrm{C}$ infections in the eastern region, including Shantou (8.2\%) and Chaozhou (2.0\%). Heterosexual (81.6\%), IDU (16.3\%), and MCT (2.0\%) were the risk factors for $\mathrm{C}$ infections. Subtype $\mathrm{G}$ was identified only in
Guangzhou, and the risk factor was heterosexual transmission.

\section{Discussion}

The predominant HIV-1 transmission route has changed with the changing socio-economic situations in China. Sexual transmission has been the main risk factor for HIV-1 infection, especially in the MSM group whose HIV-1 status remains unknown [14]. As one of regions on the forefront of the reform and opening of China, Guangdong has experienced similar trend and changes in HIV-1 risk factors [15]. We performed a comprehensive cross-sectional city-based survey in Guangdong using a molecular genotyping approach to understand 
the distribution characteristics of HIV genotypes. A total of 15 HIV-1 genotypes, including six discordant genotypes, were detected. This is more complicated than previously reported [6]. The HIV genotypic complexity in Guangdong may reflect the active mobility of people across the province.

The change of risk populations affected the distribution of HIV-1 genotypes and future trends in Guangdong. Similar to the HIV genotype survey in 2006 [6], the main prevailing HIV-1 genotypes in Guangdong remained CRF01_AE, CRF07_BC, and CRF08_BC. The proportion of CRF01_AE (including CRF55_01B and CRF59_01B) among HIV-1 infected people in Guangdong increased from $49.7 \%$ in 2006 to $53.5 \%$ in 2013 [ 6 , $16,17]$. The proportion of CRF01_AE in heterosexually infected persons remained similar to 2006 (60.7\%) and $2013(61.0 \%)$, while the prevalence in IDU decreased from $44.1 \%$ in 2006 to $31.5 \%$ in 2013 . However, the ratio of CRF01_AE in MSM unexpectedly increased from $33.3 \%$ in 2006 to $44.1 \%$ in 2013 , which was also observed in nationwide studies $[18,19]$. It is anticipated that the CRF01_AE infected cases will keep increasing if HIV infections in MSM continue growing rapidly. Similar to CRF01_AE, the proportion of CRF07_BC among HIV-1 infected people in Guangdong rose from 22.3\% in 2006 to $26.3 \%$ in 2013. The percentage of CRF07_BC increased in IDU (from $23.5 \%$ in 2006 to $35.8 \%$ in 2013), heterosexuals (from 17.9 to $21.3 \%$ in the respective years), and MSM (from 33.3 to $34.2 \%$ ) populations, which suggests that CRF07_BC would be the most likely genotype to increase in the near future. The proportion of HIV infected persons with CRF08_BC dropped drastically from $21.9 \%$ in 2006 to $8.4 \%$ in 2013 . The percentage of CRF08_BC in IDU (including heterosexual plus IDU) dropped slightly from $29.4 \%$ in 2006 to $24.9 \%$ in 2013, and increased from $3.6 \%$ in 2006 to $9.0 \%$ in 2013 in heterosexual infected persons [6]. In Guangdong, the leading risk factor for HIV infection shifted from IDU (72.2\%) in 2006 to heterosexual transmission (61.9\%) in 2013, which is the primary cause for the decrease of the proportion of CRF08_BC. However, as the proportion of CRF08_BC in the heterosexual population increased over time, close attention should be paid to the future changes of the CRF08_BC [20]. In addition, it has been suggested that the CRF07_BC and CRF08_BC have been introduced into northern Myanmar via IDU, resulting in a subtype change related epidemic in Southeast Asia [21]. The extensive prevalence of subtypes like CRF01_AE, which was originally identified in Thailand and which has been transmitted across Thailand and other southeastern Asian countries predominantly through heterosexual behavior, is notable [22, 23].

As one of the most densely urbanized areas in the world and economically developed area in Guangdong, the PRD region has frequent foreign visitors and a sizable transient population. The high mobility of people has shaped the complexity of HIV genotypes in Guangdong. It is noteworthy that the proportion of HIV discordant genotypes was relatively high and increased from $0.6 \%$ in 2006 to $8.8 \%$ in 2013, which was much higher than that of national level in $2006(2.6 \%)$ [6] or 2015 (3.8\%, personal communication). Heterosexual sex (59.2\%) has become the leading risk factor for the discordant genotypes, followed by MSM (34.4\%) and IDU (6.4\%), which is much different from that in 2006, when discordant genotypes were exclusively associated with MSM, and is consistent with other studies conducted in Guangdong $[6,9]$. The increase of discordant genotypes circulating in the province suggests that there might be an active cross-transmission of HIV-1 genotypes among the high-risk populations. This provides further indication that the risk population switch has affected HIV genotype distribution. Additionally, a sentinel survey for MSM in Guangdong showed that 21.6 and 24\% of MSM were married or had sex with women within the prior six months during the survey [24]. Therefore, considering the rapid transmission of HIV among MSM [18] and the sexual behaviors between women and MSM [25], along with the circulating CRF01_AE or the emergence of new second generation recombinants [26], the MSM group is still the great concern for HIV/AIDS interventions. More importantly, several studies also have observed the epidemic dynamics and complicated clusters of CRF01_AE in China $[19,27,28]$. With the generalization of the One Belt One Road Initiative, the communications between Asian and European countries are becoming more frequent. The burgeoning flow of people between China and global locales is increasing the challenge of infectious diseases [29], especially sexually transmitted diseases, such as HIV/AIDS, and for the introduction of new HIV recombinant forms [30].

Our updated data also reveal that Zhanjiang might be another potential epicenter in addition to Guangzhou and Shenzhen. In the city distribution pattern of HIV genotypes in Guangdong (Fig. 3), higher HIV-1 infected case numbers were found in Zhanjiang than the neighboring city of Maoming for both CRF01_AE and CRF07_BC. Especially, CRF01_AE in Zhanjiang was the highest in the west of Guangdong.

Our study has several limitations. Although the samples and the genotypes in our study accounted for 21.9 and $18.4 \%$ of all HIV infections in Guangdong in 2013, respectively, there may have been sampling bias due to the limited sample number for some risk populations and cities, for instance, IDU in Guangzhou or Yunfu. Also, since the positive ratio for PCR amplification with samples in 2013 from Chaozhou and Shanwei was low, we selected some archived samples 
from patients reported in 2014 as a supplement to those two cities.

In summary, our study clarifies the recent state of the HIV/AIDS epidemic in Guangdong. The newly-revealed regional and risk group-specific transmission patterns provide information that will be critical for developing effective interventions against HIV transmission in Guangdong.

\section{Conclusions}

Our study provides a comprehensive molecular epidemiologic dataset to understand the diversity and distribution of HIV genotypes in Guangdong, as well as to clarify the unique region- and risk group-specific transmission dynamics. The results provide critical and insightful information for more effective intervention strategies to limit HIV transmission in the future.

\section{Additional files}

Additional file 1: Table S1 Representative evaluation of genotyped samples by city in the study. (PDF $87 \mathrm{~kb}$ )

Additional file 2: Table S2 Estimated number of HIV-1 genotype in different cities and risk groups. (PDF $215 \mathrm{~kb}$ )

\section{Abbreviations}

AIDS: Acquired immunodeficiency syndrome; CRF: Circulating recombinant form; HIV: Human immunodeficiency virus; IDU: Injecting drug user; MCT: Mother to child transmission; MSM: Men who have sex with men; NA: Not available; OBOR: One Belt One Road Initiative; PRD region: Pearl River Delta region; $x^{2}$ : Chi squared test

\section{Acknowledgements}

The authors thank Dr. Ping Zhong from Shanghai Municipal Center for Disease Control and Prevention, and Dr. Tommy Lam from University of Hong Kong, for their comments on this paper.

\section{Funding}

This work was supported by the Guangdong Provincial Key Medical Discipline for AIDS Prevention and Control, and the National Science and Technology Major Project for Infectious Diseases Control and Prevention (2012ZX10004-902). The funder had no role in the design, data collection, analysis and interpretation of results in this study.

\section{Availability of data and materials}

The data are available upon request from the corresponding authors.

\section{Authors' contributions}

$X H, J Y, P L$ conceived and designed the study. PPZ, XHH performed the experiments. PPZ, XH analyzed the data and drafted the manuscript. JY, GY, $Y Q K, Y L, X F, P L$ interpreted data and provided critical reviews. All authors reviewed and approved the final manuscript.

\section{Ethics approval and consent to participate}

The study was approved by the Institutional Review Board for Medical Research of Guangdong Provincial Center for Disease Control and Prevention (No. 2016031), and informed consent from the participants was waived.

\section{Consent for publication}

Not applicable.

\section{Competing interests}

The authors declare that they have no competing interests.

\section{Publisher's Note}

Springer Nature remains neutral with regard to jurisdictional claims in published maps and institutional affiliations.

\section{Author details}

'Guangdong Provincial Institute of Public Health, Guangdong Provincial Center for Disease Control and Prevention, 160 Qunxian Road, Panyu District, Guangzhou 511430, China. ${ }^{2}$ Guangdong Provincial Center for Disease Control and Prevention, 160 Qunxian Road, Panyu District, Guangzhou 511430, China. Institute of Infection and Immunity, Henan University, Kaifeng 475000, China.

Received: 5 February 2018 Accepted: 7 February 2019

Published online: 22 February 2019

\section{References}

1. Lin P, Xu R, Zeng C, Wang Y, Li H, Lin M, Yan J. Trend and characteristics of HIV/AIDS epidemic in Guangdong province, 1986-1999. Zhonghua Liu Xing Bing Xue Za Zhi. 2001;22(3):194-7.

2. Lin $P$, Wang $Y, L i J$, Fu X, Diao L, Li Y, Lin P, Liu Y, Yan X, He Q, et al. HIV/ AIDS prevalence and control strategies in Guangdong Province. South Chin J Prev Med. 2008:34:1-5.

3. Li Y, Lin P, Wang Y, Fu X, Ye R, Li J, Feng M, Yu G, Yang F, Yan J, et al. HIV/ AIDS surveillance in Guangdong Province, 2000-2013. South Chin J Prev Med. 2015;41(2):101-6.

4. Yan J, Wang Y, Li J, Ma P, Lin P, Xing H, Diao L, Shao Y. molecular epidemiological investigation on HIV-1 epidemic strain. Chin J Public Health. 2005;21(1):90-1.

5. Zhuo L, Tang X, Chen W. HIV-1 subtype in AIDS patients in Guangdong. Inter J Epidemiol Infect Dis. 2011;38:21-5.

6. He X, Xing H, Ruan Y, Hong K, Cheng C, Hu Y, Xin R, Wei J, Feng Y, Hsi JH, et al. A comprehensive mapping of HIV-1 genotypes in various risk groups and regions across China based on a nationwide molecular epidemiologic survey. PLoS One. 2012;7(10):e47289.

7. Han Z, Xing H, Xu H, Liu S, Li Y, Ma P, Liang C, Gao K, Shao Y. molecula epidemiological study of HIV-1 positive individuals in Guangzhou from 2004-2005. Chin J AIDS STD. 2006:14:232-4.

8. Feng $T$, Zhao G, Chen $L$, Wang $X$, Shi $X$. human immunodeficiency virus type 1 strains epidemic in Shenzhen. ACTA Academiae Medicinae Sinicae. 2006;28:637-41.

9. Zhao J, Chen L, Chaillon A, Zheng C, Cai W, Yang Z, Li G, Gan Y, Wang X, Hu Y, et al. The dynamics of the HIV epidemic among men who have sex with men (MSM) from 2005 to 2012 in Shenzhen, China. Sci Rep. 2016;6:28703.

10. Cheng $C L$, Feng $Y$, He $X$, Lin $P$, Liang SJ, Yi ZQ, He JM, Hu YY, Xing H, Fan $Y$, et al. Genetic characteristics of HIV-1 CRF01_AE strains in four provinces, southern China. Zhonghua Liu Xing Bing Xue Za Zhi. 2009;30(7):720-5.

11. Hall TA. BioEdit: a user-friendly biological sequence alignment editor and analysis program for windows 95/98/NT. Nucl Acids Symp Ser. 1999:41:95-8.

12. Tamura K, Peterson D, Peterson N, Stecher G, Nei M, Kumar S. MEGA5: molecular evolutionary genetics analysis using maximum likelihood, evolutionary distance, and maximum parsimony methods. Mol Biol Evol. 2011;28(10):2731-9.

13. Siepel AC, Halpern AL, Macken C, Korber BT. A computer program designed to screen rapidly for HIV type 1 intersubtype recombinant sequences. AIDS Res Hum Retrovir. 1995;11(11):1413-6.

14. Tang Q, Lu H. discussion on epidemic status and control strategies of HIV/ AIDS. Fudan Univ J Med Sci. 2017;44(6):744-51.

15. Chen S, Cai W, He J, Vidal N, Lai C, Guo W, He H, Chen X, Fu L, Peeters M, et al. Molecular epidemiology of human immunodeficiency virus type 1 in Guangdong province of southern China. PLoS One. 2012;7(11):e48747.

16. Wan ZY, Xing H, Li J, Wang Y, Diao LM, Ma PF, Lin P, Jiang SL, Shao YM. Subtype and sequence analysis of human immunodeficiency virus type-1 strains in Guangdong province. Zhonghua Yu Fang Yi Xue Za Zhi. 2006; 40(5):344-7.

17. Zhang W, Han X, An M, Zhao B, Hu Q, Chu Z, Xu J, Cai W, Chen X, Fu J, et al. Identification and characterization of a novel HIV-1 circulating recombinant form (CRF59_01B) identified among men-who-have-sex-withmen in China. PLoS One. 2014;9(6):e99693.

18. Li X, Gao R, Zhu K, Wei F, Fang K, Li W, Song Y, Ge Y, Ji Y, Zhong P, et al. Genetic transmission networks reveal the transmission patterns of HIV-1 CRF01_AE in China. Sex Transm Infect. 2018;94(2):111-116. 
19. Zeng H, Li T, Wang Y, Sun B, Yang R. The epidemic dynamics of four major lineages of HIV-1 CRF01_AE strains after their introduction into China. AIDS Res Hum Retrovir. 2016;32(5):420-6.

20. Feng Y, Takebe Y, Wei H, He X, Hsi JH, Li Z, Xing H, Ruan Y, Yang Y, Li F, et al. Geographic origin and evolutionary history of China's two predominant HIV-1 circulating recombinant forms, CRF07_BC and CRF08_BC. Sci Rep. 2016;6:19279

21. Chen $X$, Ye M, Pang W, Smith DM, Zhang C, Zheng YT. First appearance of HIV-1 CRF07_BC and CRF08_BC outside China. AIDS Res Hum Retrovir. 2017; 33(1):74-6.

22. Zhang Y, Lu L, Ba L, Liu L, Yang L, Jia M, Wang H, Fang Q, Shi Y, Yan W, et al. Dominance of HIV-1 subtype CRF01_AE in sexually acquired cases leads to a new epidemic in Yunnan province of China. PLoS Med. 2006;3(11):e443.

23. Gao F, Robertson DL, Morrison SG, Hui H, Craig S, Decker J, Fultz PN, Girard M, Shaw GM, Hahn BH, et al. The heterosexual human immunodeficiency virus type 1 epidemic in Thailand is caused by an intersubtype (a/E) recombinant of African origin. J Virol. 1996;70(10):7013-29.

24. Fu X, Lin $P$, Wang $Y, L i J, Y u G, L i$ Y. prevalence of human immnodeficiency virus and syphilis among MSM in Guangdong from 2009 to 2013. Chin Prev Med. 2014;15(5):169-72.

25. Li X, Zhang B, Wang J, Li Y, Yu P, Liu M, Liu X. Sexual health status of women who have regular sexual relations with men who have sex with men in mainland China. BMC Public Health. 2017;17(1):168.

26. Li X, Hao M, Hao Y, Ye J, Xin R, Lu H. A unique second-generation recombinant form of HIV-1 identified among men who have sex with men population in Beijing, China. AIDS Res Hum Retrovir. 2017:33(10):1070-6.

27. Wang X, He X, Zhong P, Liu Y, Gui T, Jia D, Li H, Wu J, Yan J, Kang D, et al. Phylodynamics of major CRF01_AE epidemic clusters circulating in mainland of China. Sci Rep. 2017;7(1):6330

28. Feng $Y$, He $X, H$ si JH, Li F, Li X, Wang $Q$, Ruan $Y$, Xing H, Lam TT, Pybus OG, et al. The rapidly expanding CRF01_AE epidemic in China is driven by multiple lineages of HIV-1 viruses introduced in the 1990s. AIDS. 2013; 27(11):1793-802

29. Qian MB, Abela-Ridder B, Wu WP, Zhou XN. Combating echinococcosis in China: strengthening the research and development. Infect Dis Poverty. 2017:6(1):161.

30. Wu Y, Ren X, Yin D, Wang H, Wan Z, Li X, Hu G, Tang S. Characterization of a novel HIV-1 unique recombinant form between CRF07_BC and CRF55 01B in men who have sex with men in Guangzhou, China. PLoS One. 2017; 12(4):e0175770.

Ready to submit your research? Choose BMC and benefit from:

- fast, convenient online submission

- thorough peer review by experienced researchers in your field

- rapid publication on acceptance

- support for research data, including large and complex data types

- gold Open Access which fosters wider collaboration and increased citations

- maximum visibility for your research: over $100 \mathrm{M}$ website views per year

At $\mathrm{BMC}$, research is always in progress.

Learn more biomedcentral.com/submissions 